\title{
会i- \\ Efectos del mercado laboral sobre el sector financiero en la OCDE
}

\author{
Guillermo Peña Blasco \\ Departamento de Estructura e Historia Económica \\ Y ECONOMÍA PÚBLICA \\ FACULTAD DE ECONOMÍA Y EMPRESA \\ UNIVERSIDAD DE ZARAGOZA \\ gpena@unizar.es
}

Resumen:

Introducción: este trabajo estudia el impacto del desempleo sobre el desarrollo financiero a través de una relación no lineal. La literatura ha estudiado el tema del impacto del desarrollo financiero en la tasa de desempleo, pero en cambio el análisis de los efectos del desempleo en el desarrollo financiero casi no ha sido estudiado.

Material y métodos: utilizamos un panel no balanceado con los 35 países miembros de la OCDE de 1996 a 2014, realizando un análisis descriptivo y estimando modelos econométricos dinámicos de datos de panel a través de los métodos GMM System y en diferencias.

Resultados: observamos un impacto significativo y negativo del desempleo sobre los costes bancarios, a través de una relación no lineal de proporcionalidad inversa. 
Discusión: las estimaciones corroboran la relación no lineal que ha sido observada en el análisis descriptivo. Una reforma del sector financiero que desvinculara los efectos del desempleo sobre el desarrollo financiero permitiría reducir los efectos no deseables de los vínculos entre economía real y financiera.

Palabras clave: Mercado laboral, sector financiero, desempleo, desarrollo financiero.

\section{Effects of the labour market on the financial sector at the OECD}

\section{Abstract:}

Introduction: this paper analyzes the impact of unemployment on financial development through a non-linear relationship. Some papers have analyzed the impact of financial development on unemployment, but only a few papers have studied the inverse effect, namely the impact of unemployment on financial development.

Material and methods: a non-balanced panel is used, covering all the 34 members of the OECD from 1996 to 2014, and a descriptive analysis performed. Econometric models for dynamic panel data are subsequently estimated using System GMM and Difference GMM methods.

Results: a significant negative impact of unemployment on banking costs through a non-linear relationship of inverse proportionality is found.

Discussion: the non-linear relationship revealed by a descriptive analysis is corroborated by these estimations. A reform of the financial sector delinking the effects of unemployment from the financial sector would avoid the undesirable effects of the links between the financial economy and the real economy.

Key words: Labour market, financial sector, unemployment, financial development. 


\section{Efectos del mercado laboral sobre el sector financiero en la OCDE}

Guillermo

Peña Blasco

https://doi.org/10.26754/ojs_ais/ais.2019393240

Recibido: 28/02/2017

Aceptado: 14/05/2018

\section{INTRODUCCIÓN}

En la actualidad hay consenso en que el mal funcionamiento del sector financiero genera crisis económicas (FMI, 2010). Sin embargo, únicamente en los últimos años ha crecido el interés por el estudio del sector financiero y laboral. El interés en el primero se ha incrementado debido al estallido en 2007 de la Crisis Financiera Global (CFG), y se ha prestado mayor atención también al mercado laboral debido a los efectos de la Gran Recesión (GR) que se originó a raíz de la CFG.

Sin embargo, a pesar de la adopción de medidas de regulación financiera y bancaria o de gravamen de impuestos Pigouvianos con el objetivo de evitar un excesivo desarrollo financiero, este sector de la economía sigue estando sobre-dimensionado, debiendo mejorar su eficiencia. En este trabajo, aparte del enfoque tradicional de considerar el impacto del desarrollo financiero sobre el desempleo, estudiamos el impacto del desempleo sobre el desarrollo financiero, sugiriendo que uno de los posibles determinantes del desarrollo financiero insostenible de los últimos años es el mercado laboral, en concreto, la tasa de desempleo, al igual que otros autores han encontrado la desigualdad económica como determinante de este ineficiente desarrollo financiero.

La Real Academia de la Lengua Española (RAE) define mercado como el "conjunto de actividades realizadas libremente 
por los agentes económicos sin intervención del poder público", mientras que laboral es "perteneciente o relativo al trabajo, en su aspecto económico, jurídico y social." El tópico del mercado laboral, dentro de la Economía laboral, engloba diversos y variados temas. Sin embargo, en este trabajo nos centramos en un tema tan poco tratado por esta rama del conocimiento como es la relación entre la tasa de desempleo y el desarrollo financiero. En concreto, nos fijamos en el impacto de esta tasa sobre la eficiencia bancaria, medida como los costes bancarios sobre el total de activos de las entidades, para la OCDE durante los años 1996 a 2014. Consideramos que este análisis es de vital importancia para comprender mejor las interacciones entre mercado laboral y sector financiero.

Este trabajo consta de seis secciones. A continuación de la introducción comienza la segunda sección, en la que se realiza un análisis descriptivo de algunos hechos estilizados. En la tercera sección se formula la hipótesis teórica del manuscrito, se analiza teóricamente la relación y se revisa la literatura sobre el tópico. La cuarta sección expone el material y los métodos utilizados. La quinta sección muestra los resultados y realiza una breve discusión. Por último, la sección sexta concluye.

\section{ANÁLISIS DESCRIPTIVO}

Mientras que durante los años sesenta y principios de los ochenta del siglo XX el desempleo se incrementó en todos los países de la OCDE, desde los principios de los años ochenta la tendencia de la tasa de desempleo de estos países diverge, habiendo países en los que crece, se estabiliza, o decrece (Belot y Van Ours, 2001). En la Figura 1 se puede apreciar la evolución de la tasa de paro y de los costes bancarios sobre el total de activos en la OCDE durante los años 2002 a 2013. Se observa que la tasa de paro en los años previos a la Gran Recesión disminuyó para la media de la OCDE, se incrementó a partir de 2008 y llegó al máximo de porcentaje de desempleo en $2010^{1}$.

${ }^{1}$ Se ha utilizado esta serie debido a la disponibilidad de datos: son los únicos años de la muestra para los que se tiene información sobre los costes para la integridad de los países de la OCDE. 
La evolución de los costes sigue la tendencia aproximadamente contraria: mientras que de 2002 a 2007 los costes alcanzan una ratio en torno a $2 \%$, cuando la tasa de desempleo alcanza un máximo de $7 \%$, en cambio, en el periodo en el que la tasa de desempleo llega a niveles del $8 \%$, los costes se sitúan en torno a 1,6\%. Se aprecia, por lo tanto, una relación de signo negativo entre ambas variables.

\section{Figura 1}

Evolución de la tasa de desempleo de 2002 a 2013 para la media de la OCDE

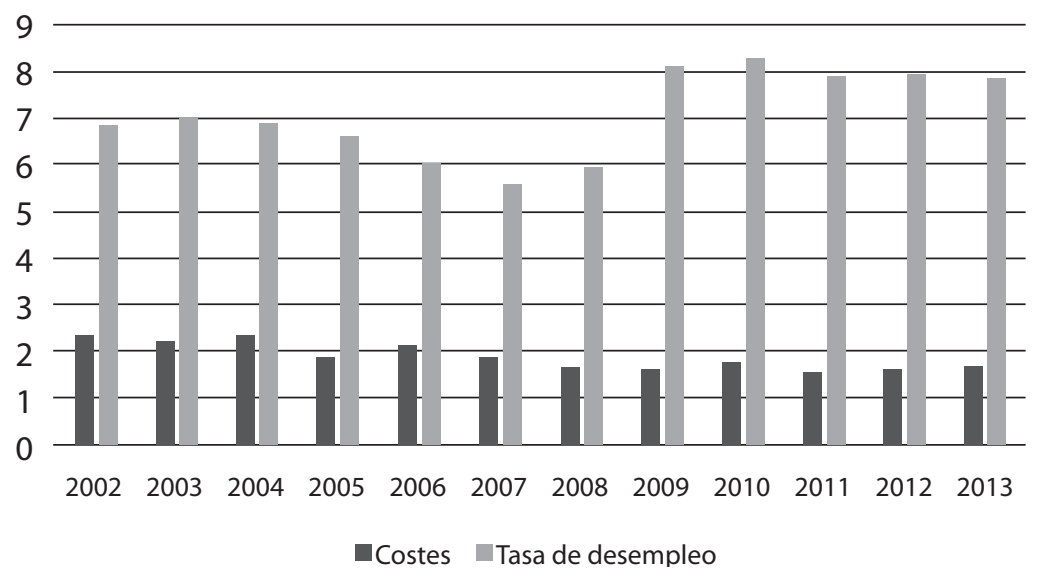

Fuente: Banco Mundial (2017)

La Figura 2 nos muestra la relación entre el coste de los servicios financieros, utilizando el mismo indicador que en la Figura 1, y la tasa de paro en la OCDE ${ }^{2}$ durante el año 2014. Se puede observar en el gráfico una ligera relación negativa con forma de "L" curva en los datos. Grecia y España son unos outliers en cuanto a tasa de desempleo (con una tasa de paro del $26 \%$ y $24 \%$, respectivamente), mientras que Hungría es un dato atípico en cuanto a porcentaje de costes de servicios financieros, superando una ratio de $4 \%$. Se puede observar que las tasas de

${ }^{2}$ Se han utilizado los datos de todos los países de la OCDE menos de Irlanda y de Bélgica, para los que no hay datos en la muestra. 
desempleo en varios países de la OCDE son todavía relativamente altas. Una posible explicación que es aceptada por la mayor parte de la comunidad científica, aunque no está universalmente aceptada, considera que estos altos niveles de desempleo se deben principalmente a las altas rigideces en el mercado de trabajo (salarios no flexibles, poder de los sindicatos o patronal, tipo de instituciones, etc.) (Nickell et al., 2005).

\section{Figura 2}

\section{Relación entre costes bancarios y tasa de paro en la OCDE en 2014}

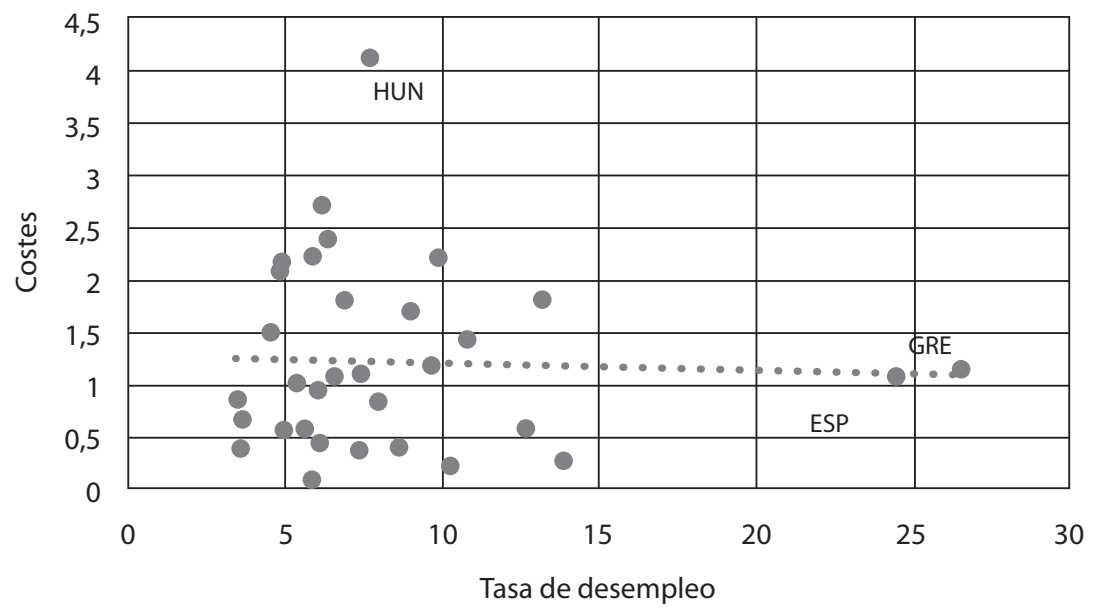

Fuente: OCDE (2017). HUN: Hungría, GRE: Grecia, ESP: España

Algunos autores discuten una serie de políticas para combatir estas rigideces (Blanchard et al., 2014). Los autores se centran en dos capacidades fundamentales que la economía tiene que poseer para superarlas: conseguir "micro-flexibilidad", que permite redistribuir los trabajadores a empleos que favorecen el crecimiento económico; y la "macro-flexibilidad", que permite ajustar la economía a los shocks macroeconómicos. Esta capacidad de la economía para superar las rigideces depende fundamentalmente de cómo estén diseñadas sus instituciones, una variable que cambia poco a corto y largo plazo. 
La ratio de costes sobre total de activos bancarios se considera un indicador de eficiencia bancaria, y de desarrollo financiero, de manera que una menor ratio significa una mayor eficiencia bancaria y un desarrollo financiero más sostenible. El "desarrollo financiero" se define como la facilidad con la que una persona, física o jurídica, puede obtener financiación (Rajan y Zingales, 2003). A su vez, definimos desarrollo financiero sostenible como aquel desarrollo financiero más competitivo, con menores costes y beneficios y más eficiente. El desarrollo financiero se puede medir de diversas formas, como el porcentaje de créditos sobre el total del PIB, el porcentaje de primas de seguros, el porcentaje de valor añadido que representa el sector financiero sobre el total de la economía, o como la ratio de costes bancarios sobre el total de activos de las entidades financieras, indicador de eficiencia que vamos a utilizar en el trabajo.

\section{RELACIÓN TEÓRICA ENTRE SECTOR FINANCIERO Y ECONOMÍA REAL Y FORMULACIÓN DE HIPÓTESIS}

Perugini et al. (2015) encuentran que la creencia generalizada de que la distribución de la renta no es relevante en la estabilidad macroeconómica (incluido el desarrollo financiero) necesita ser reconsiderada y que, más que considerar factores de política monetaria y reformas en la regulación bancaria para hacer más robusto el sector financiero, los políticos deben prestar más atención a los cambios en los patrones de distribución de la renta. En este artículo consideramos cómo impacta el desempleo en el desarrollo financiero, pudiendo considerar el desempleo como indicador de desigualdad o pobreza relativa, ya que el desempleo implica no obtener unos ingresos o rentas laborales que los empleados sí que obtienen, y se genera así una asimetría económica entre empleados y desempleados, teniendo estos últimos una renta más baja, ceteris paribus.

El mecanismo de transmisión del desempleo al mercado laboral sería el siguiente, destacando dos efectos sobre los costes bancarios del mismo signo. En primer lugar, hay que considerar los efectos a nivel microeconómico, considerando tanto la oferta de trabajo como la demanda de servicios financieros, es decir, el lado del hogar. Hay que tener en cuenta que los usuarios más frecuentes de servicios financieros son personas de renta alta (Huizinga 
2002). De esta forma, pasar a formar parte de la población desempleada llevará al desempleado a ver disminuir su renta (ya que el subsidio que recibe es menor que el salario que percibía), lo que le llevará a utilizar con menor frecuencia los servicios financieros, contrayendo la demanda de servicios financieros y contribuyendo a que la empresa incurra en menores costes y a una mejora en eficiencia. Por otro lado, también se reducirán los costes debido a que el sector bancario será más reticente a concederle servicios financieros como préstamos o hipotecas debido al riesgo de impago. Además, se pueden considerar que, si una persona pasa del empleo al desempleo, ello implicará un proceso de adaptación en el que al principio la persona en cuestión seguirá manteniendo al menos parte de su nivel de vida, lo que le llevará al principio a seguir utilizando con frecuencia los servicios financieros, pero cada vez en menor medida conforme se vaya adaptando a la situación actual de desempleo. Esta paulatina adaptación explicaría una especificación no lineal en la relación.

En segundo lugar, consideramos los efectos a nivel macroeconómico, teniendo en cuenta tanto la demanda de trabajo como la oferta de servicios financieros, es decir, el lado de las entidades financieras. La contracción de la demanda lleva a una restricción en la oferta, por medio de una amortización de los puestos de trabajo con baja productividad por parte de la banca, reduciéndose los costes salariales de las entidades financieras y disminuyendo así la ratio de costes sobre los ingresos, mejorando, por lo tanto, su eficiencia. Además, esta mejora en eficiencia será también cada vez menos pronunciada, debido a la ley de rendimientos decrecientes del trabajo.

Por lo tanto, la hipótesis que sostenemos en este artículo es que el desempleo impacta negativamente sobre los costes bancarios, confirmando la pendiente negativa de la tendencia de la Figura 2. De hecho, consideramos que el desempleo impacta de forma positiva sobre la eficiencia bancaria, pero que el impacto conforme aumenta el desempleo es cada vez menor, de forma similar que ocurre en la no monotonicidad del PIB per cápita respecto del crédito (Cecchetti y Kharroubi, 2012). Por lo tanto, la hipótesis a considerar es:

\section{H1: El desempleo impacta sobre la ratio de costes bancarios por activos siguiendo una proporcionalidad inversa, de forma que un}




\section{incremento en la tasa de desempleo mejora la eficiencia bancaria, favoreciendo, por lo tanto, un desarrollo financiero más sostenible.}

En cuanto a la relación contraria entre los servicios financieros y el mercado laboral, Acemoglu (2001) fue uno de los pioneros en estudiar cómo las restricciones bancarias pueden afectar al comportamiento del desempleo, concluyendo que las imperfecciones de los mercados de crédito incrementan los niveles de desempleo y su persistencia. Algunos autores confirman esta teoría, obteniendo empíricamente que el desarrollo financiero, medido por el crédito privado, impacta negativamente sobre la tasa de desempleo (Ogbeide et al., 2015), mientras que otros han mostrado que personas que vivían en distritos con mayor número de sucursales bancarias eran significativamente menos proclives a ser autónomos (Dehejia y Gupta, 2016). Esto se debe a que el buen funcionamiento del sector financiero lleva a una eficiencia económica que puede promover una respuesta emprendedora competente a las oportunidades de negocios (Schumpeter, 1911). Por lo tanto, las expectativas de que se produzca un incremento del crédito en el sector privado llevarán a una caída en el desempleo debido a la expansión empresarial (Ogbeide et al., 2015).

Este hecho tendrá que ser tenido en cuenta a la hora de evitar problemas de endogeneidad. Sorprende que, mientras el impacto del sector financiero sobre la economía real ha sido ampliamente estudiado, encontrando que el desarrollo financiero favorece la economía o reduce el desempleo y la pobreza (Acemoglu, 2001; Dromel et al., 2010; Ibrahim y Aliero, 2012; Aliero at al., 2013), se ha estudiado poco la relación en el sentido contrario, si acaso considerando únicamente el impacto de la renta per cápita (Perugini et al., 2015).

Este trabajo va a estudiar el impacto de la tasa de desempleo sobre los costes bancarios, como indicador de desarrollo financiero, considerando que unos menores costes bancarios significan una mayor madurez del sistema bancario, explicando este indicador por medio de las principales variables encontradas en la literatura. Perugini et al. (2015) consideran varios determinantes del desarrollo del sector financiero por medio de la aplicación de técnicas de GMM System, que son las más habituales a la hora de investigar los determinantes del desarrollo financiero, debido 
a los problemas de endogeneidad y recursividad de la variable dependiente. Como variables explicativas utilizan la capitalización bancaria, obteniendo un signo positivo y significativo en sus estimaciones. Se podría considerar que una economía con un sector financiero más capitalizado conseguirá alcanzar un mayor desarrollo financiero debido a la esterilización de la política monetaria, que afecta menos a los bancos. También consideran indicadores de política monetaria, como el tipo de interés real o la masa monetaria, sin embargo, obtienen resultados no significativos estadísticamente.

Encuentran un impacto positivo y significativo de la desigualdad de la renta en la variable independiente, medida por el porcentaje de población por encima del primer percentil de renta, además de obtener significatividad positiva del coeficiente de la renta per cápita. Esto se puede interpretar como que un crecimiento mayor de la renta lleva a su vez a un mayor desarrollo financiero, lo que puede provocar la creación un círculo vicioso, ya que, si se produce un mal funcionamiento del sector bancario, que genera un desarrollo financiero diferente del óptimo (por ejemplo, al no controlar el grado de capitalización bancaria), esta ineficiencia de las entidades financieras se puede llegar a agravar e incluso, en último término, a desencadenar crisis económicas (Peña, 2017).

Las variables poblaciones también suelen ser utilizadas para explicar el desarrollo financiero. Por ejemplo, una mayor densidad poblacional podría generar un mayor desarrollo económico porque, si en una región está más concentrada la población, habrá un mayor acceso a los servicios financieros y por lo tanto se puede esperar un signo positivo del coeficiente. Sin embargo, la evidencia empírica para esta variable es mixta: algunos consideran u obtienen un impacto positivo (Demirgüç-Kunt et al., 2011) o negativo o nulo (Allen et al., 2014).

Otra variable a tener en cuenta debido al posible crowding-out (efecto desplazamiento) entre crédito del sector privado y del sector público, es la deuda privada o el déficit público, esperando un efecto negativo de esta variable varios autores (Caballero y Krishnamurthy, 2004; Christensen, 2005 y Ayadi et al., 2013). Finalmente, se suele utilizar muchas veces la variable inflación como indicador de estabilidad económica, obteniendo resultados que indican 
un impacto habitualmente positivo (Boyd et al., 2001; Detragiache et al., 2005; Asongu, 2014 y Bahadir y Valev, 2015).

\section{FUENTES ESTADÍSTICAS Y METODOLOGÍA}

La Tabla 1 muestra las variables explicativas utilizadas para explicar la variable dependiente, junto con el signo esperado, la fuente y las referencias encontradas en la literatura. Como dependiente utilizamos la variable costes, obtenida del Banco Mundial (2017) y que representa la ratio de costes totales bancarios sobre el total de activos, en porcentaje, reflejando la estructura de costes de las entidades, como indicador de desarrollo financiero y eficiencia bancaria.

La razón de otorgar un signo esperado negativo al coeficiente del desempleo radica en el razonamiento teórico aportado en la sección anterior y en los hechos estilizados basados en la observación de la relación decreciente entre países de la Figura 2. Para obtener una estimación más rigurosa y precisa del desarrollo financiero y de la influencia del desempleo sobre esta variable, se van a incluir las variables explicativas de desarrollo económico más relevantes y sobre las que hay más consenso.

\section{Tabla 1}

Variables y signo esperado del impacto sobre los costes bancarios

\begin{tabular}{|c|c|c|c|c|}
\hline Variable & Definición & Fuente & Signo & Referencias \\
\hline precios & $\begin{array}{c}\text { Índice de precios tomando } 2010 \\
\text { como año base }\end{array}$ & OCDE & $(+/-)$ & $\begin{array}{c}(-): \text { Bahadir and Va- } \\
\text { lev (2015), (+): Khan } \\
\text { et al. (2006) }\end{array}$ \\
\hline superávit & $\begin{array}{c}\text { Superávit total del gobierno sobre } \\
\text { el PIB }\end{array}$ & $\begin{array}{c}\text { Banco } \\
\text { Mundial }\end{array}$ & $(+)$ & Ayadi et al. (2013) \\
\hline densidad & $\begin{array}{r}\text { Población de hecho de un país } \\
\text { dividido por su superficie }\end{array}$ & $\begin{array}{c}\text { Banco } \\
\text { Mundial }\end{array}$ & $(+/-/ 0)$ & $\begin{array}{c}(+) \text { : Demirgüç-Kunt } \\
\text { et al. (2011), (-/0): } \\
\text { Allen et al., (2014) }\end{array}$ \\
\hline pibpc1 & PIB per cápita retardado un periodo & OCDE & $(+)$ & Allen et al. (2014) \\
\hline pibpc2 & $\begin{array}{r}\text { PIB per cápita al cuadrado retarda- } \\
\text { do un periodo }\end{array}$ & OCDE & $(+)$ & $\begin{array}{c}\text { Cecchetti and Khar- } \\
\text { roubi (2012) }\end{array}$ \\
\hline $\begin{array}{r}\text { recdes- } \\
\text { empleo }\end{array}$ & $\begin{array}{c}\text { Recíproco o inverso de la tasa de } \\
\text { desempleo retardado un periodo }\end{array}$ & OCDE & $(+)$ & Los autores \\
\hline
\end{tabular}


La matriz de correlaciones de las variables explicativas se expone en la Tabla 2, observándose que ningún valor llega a 0,5, quedando por debajo de valores indicativos de un posible riesgo de multicolinealidad.

\section{Tabla 2}

Matriz de correlación de las variables explicativas

\begin{tabular}{|c|c|c|c|c|c|}
\hline & precios & superávit & densidad & pibpc & desempleo \\
\hline precios & 1 & & & & \\
\hline superávit & $-0,2208$ & 1 & & & \\
\hline densidad & 0,1156 & $-0,1921$ & 1 & & \\
\hline pibpc & 0,3410 & 0,1920 & 0,1745 & 1 & \\
\hline desempleo & 0.1435 & -0.4542 & $-0,0777$ & $-0,3922$ & 1 \\
\hline
\end{tabular}

Nos basamos en un panel no balanceado compuesto por todos los países miembros de la OCDE (35), durante el periodo de 1996 a 2014. El resumen de las principales características de las variables se presenta en la Tabla 3.

\section{Tabla 3}

Principales características de las variables

\begin{tabular}{|c|c|c|c|c|c|}
\hline Variable & $\begin{array}{c}\text { Número de } \\
\text { observaciones }\end{array}$ & Media & $\begin{array}{c}\text { Desviación } \\
\text { típica }\end{array}$ & Mínimo & Máximo \\
\hline costes & 661 & 2,1262 & 1,6255 & 0,0409 & 14.6112 \\
\hline precios & 473 & 92,4946 & 13,0523 & 59,6098 & 116.8873 \\
\hline superávit & 636 & $-1,9261$ & 4.4742 & -32.1181 & 18.6959 \\
\hline densidad & 644 & 0.0001 & 0.0001 & $2.37 \mathrm{E}-06$ & 0.0005 \\
\hline pibpc & 665 & 29575,8 & 13600,77 & 5796,768 & 100052,8 \\
\hline desempleo & 565 & 7,6351 & 3.9210 & 2,0583 & 27.46715 \\
\hline
\end{tabular}

En cuanto al método utilizado, la forma funcional se basa en el GMM en dos etapas, siguiendo a Huang (2010). La utilización de un modelo dinámico se basa en asumir que el desarrollo financiero del periodo previo afecta al periodo actual, 
una hipótesis que puede ser fácilmente comprobada por la presencia de significatividad en el coeficiente asociado al retardo temporal de la variable dependiente y presencia a su vez de buenas propiedades econométricas. Para obtener unos resultados robustos al método utilizado, estimamos por el método de GMM en diferencias desarrollado por Arellano y Bond (1991) para corregir los sesgos de endogeneidad en las variables explicativas y el GMM System desarrollado por Arellano y Bover (1995) y Blundell y Bond (1998) para mejorar la eficiencia del estimador GMM:

$$
y_{i, t}=\gamma y_{i, t-1}+\beta X_{i t}+\beta_{T} T_{i t-1}+\beta^{\prime} X_{i t-1}^{\prime}+\varepsilon_{i t},
$$

La variable $y_{i, t}$ es la variable dependiente y representa el desarrollo financiero, medido a través de la variable costes, para el país $i$ en el periodo $t$. El primer retardo de la variable dependiente es $y_{i, t-1}$, y $\gamma$ es su coeficiente. El vector de variables exógenas es $X_{i t}$, la variable objetivo es $T_{i t-1}$, que es recdesempleo, variable retardada un periodo para evitar endogeneidad (Blundell y Bond, 2000 y Peña, 2017) y $X_{i t-1}^{\prime}$ es el vector del resto de variables explicativas retardadas también un periodo para eliminar la potencial endogeneidad vista en la sección anterior. Los coeficientes de estas variables son $\beta, \beta_{T}$ y $\beta^{\prime}$, respectivamente, y $\varepsilon_{i t}$ es la perturbación aleatoria.

\section{RESULTADOS, DISCUSIÓN Y MEDIDAS DE POLÍTICA}

La metodología utilizada a la hora de estimar los modelos econométricos ha sido la siguiente. En primer lugar, se ha estimado un modelo estático de panel simple siguiendo la metodología de efectos fijos, sin embargo, parecía haber efectos no observados que no habían sido tratados debido al bajo grado de explicación de la variable dependiente a través de las variables independientes. Esto se ha comprobado al obtener un elevado coeficiente de la constante y un bajo nivel de $\mathrm{R} 2$.

Por lo tanto, teniendo en cuenta la dinamicidad y recursividad de las variables dependientes que mencionábamos previamente y atendiendo a los modelos más frecuentes a la hora de estudiar el desarrollo financiero, se ha modificado la especificación de los modelos para tener en cuenta los efectos 
no observados y se han estimado dos modelos econométricos, uno en GMM System y el otro en GMM en diferencias, obteniendo las perturbaciones a través de un estimador robusto Huber/White/Sandwich, que es un estimador robusto en el que se corrige el sesgo y se calcula por medio de estimadores de la matriz de varianzas en dos etapas de GMM. Además, la matriz de varianzas y covarianzas de los estimadores de los paneles dinámicos se estima en dos etapas (Windmeijer, 2005). A partir de estos dos modelos iniciales, una vez que se han superado los contrastes de identificación (Sargan) y de incorrelación (Arellano-Bond), se eliminan consecutivamente las variables redundantes no significativas, obteniendo los modelos definitivos.

Los resultados de los modelos con propiedades econométricas deseables se pueden apreciar en la Tabla 3. Los modelos a evaluar van a ser los definitivos, ya que los iniciales sólo se estiman para obtener aquellos. Se observan cuatro variables explicativas cuyos coeficientes son significativos: el retardo de la variable dependiente (lo que permite comprobar la existencia de dinamicidad de las variables dependientes), la variable precios (corrobora la importancia de la política monetaria para evitar el excesivo crecimiento del sector financiero, a través del control de la inflación) y las dos variables que reflejan crecimiento económico. Además, en la variable de interés se encuentra un impacto no lineal del desempleo sobre los costes bancarios, de proporcionalidad inversa, que es significativo y robusto a la metodología utilizada. 


\section{Tabla 3}

\section{Resultados obtenidos}

\begin{tabular}{|c|c|c|c|c|c|c|c|c|}
\hline \multirow{2}{*}{$\begin{array}{c}\text { Variable depen- } \\
\text { diente: costes } \\
\begin{array}{c}\text { Variables explica- } \\
\text { tivas }\end{array} \\
\end{array}$} & \multicolumn{2}{|c|}{$\begin{array}{l}\text { Modelo 1: GMM } \\
\text { System inicial }\end{array}$} & \multicolumn{2}{|c|}{$\begin{array}{l}\text { Modelo 2: GMM } \\
\text { System definitivo }\end{array}$} & \multicolumn{2}{|c|}{$\begin{array}{l}\text { Modelo 3: GMM en } \\
\text { diferencias inicial }\end{array}$} & \multicolumn{2}{|c|}{$\begin{array}{c}\begin{array}{c}\text { Modelo 4: GMM } \\
\text { en diferencias } \\
\text { definitivo }\end{array} \\
\end{array}$} \\
\hline & Coef. & Valor $p$ & Coef. & Valor $p$ & Coef. & Valor $p$ & Coef. & Valor $p$ \\
\hline $\begin{array}{c}\text { Retardo (t-1) va- } \\
\text { riable dependiente }\end{array}$ & $0,6445^{\star \star \star}$ & 0,0000 & $0,6625^{\star \star \star}$ & 0,0000 & $0,1772^{\star}$ & 0,0830 & $0,1926^{\star}$ & 0,0740 \\
\hline precios & 0,0076 & 0,1790 & $0,0041^{\star \star \star}$ & 0,0080 & $-0,0135$ & 0,2370 & $-0,0109$ & 0,3110 \\
\hline superávit & $-0,0181$ & 0,4250 & & & $-0,0007$ & 0,9730 & $-0,0039$ & 0,8330 \\
\hline densidad & $-1330,924$ & 0,5530 & & & 21926,0800 & 0,5360 & & \\
\hline pibpc2 & 0,0000 & 0,7690 & & & 0,0000 & 0,1350 & $0,0000^{\star \star}$ & 0,0450 \\
\hline pibpc1 & 0,0000 & 0,6560 & & & $-0,0001^{\star \star}$ & 0,0180 & $-0,0001^{\star \star}$ & 0,0120 \\
\hline recdesempleo & 3,1055 & 0,1720 & $2,1697^{\star \star}$ & 0,0110 & 1,6131 & 0,1850 & $1,7103^{*}$ & 0,0690 \\
\hline Sargan (valor p) & \multicolumn{2}{|c|}{0,9416} & \multicolumn{2}{|c|}{0,8146} & \multicolumn{2}{|c|}{0,1380} & \multicolumn{2}{|c|}{0,1394} \\
\hline $\begin{array}{c}\text { Arellano-Bond (valor } \\
\text { p, 1er, } 2^{\circ} \text { orden) }\end{array}$ & 0,0009 & 0,3803 & 0,0009 & 0,3764 & 0,0038 & 0,8662 & 0,0034 & 0,9441 \\
\hline No Observaciones & \multicolumn{2}{|c|}{382} & \multicolumn{2}{|c|}{392} & \multicolumn{2}{|c|}{353} & \multicolumn{2}{|c|}{363} \\
\hline No Instrumentos & \multicolumn{2}{|l|}{40} & \multicolumn{2}{|c|}{36} & \multicolumn{2}{|l|}{23} & \multicolumn{2}{|c|}{22} \\
\hline
\end{tabular}

Nota: ***: significatividad al 99\%, **: significatividad al 95\%, **: significatividad al 90\%, coef.: coeficiente

En la Tabla 4 se puede apreciar el signo obtenido en todas las variables y la comparación con el signo esperado. Se puede observar que todas las variables cumplen con el signo esperado menos el coeficiente de la variable pibpc1 en el Modelo 4, teniendo un signo negativo que puede interpretarse como que cuanto más desarrollado económicamente está un país, mayor es su eficiencia bancaria. 


\section{Tabla 4}

Tabla resumen de efectos obtenidos y esperados sobre los costes

\begin{tabular}{|c|c|c|c|}
\hline \multirow{2}{*}{ Método } & Modelo 2 & Modelo 4 & \multirow{2}{*}{ Signo esperado } \\
\cline { 2 - 4 } & GMM System & GMM en diferencias & \\
\hline retardo & $(+)$ & $(+)$ & $(+)$ \\
\hline precios & $(+)$ & & $(+/-)$ \\
\hline superávit & & & $(+)$ \\
\hline densidad & & & $(+/-/ 0)$ \\
\hline pibpc2 & & $(+)$ & $(+)$ \\
\hline pibpc1 & & $(-)$ & $(+)$ \\
\hline desempleo1 & & & $(-)$ \\
\hline recdesempleo & $(+)$ & $(+)$ & $(+)$ \\
\hline
\end{tabular}

Ciñéndonos a nuestras variables objetivo, se observa la significatividad estadística y económica del impacto negativo del desempleo sobre el desarrollo financiero, a través de una relación no lineal, en concreto de proporcionalidad inversa, confirmando la Hipótesis 1 de la tercera sección. Se ha utilizado esta especificación de la variable de interés en este artículo debido a la relación encontrada observando el cambio a lo largo del tiempo de la relación entre el desempleo y el porcentaje de valor añadido del sector financiero sobre el total en un mismo país, habiéndose obtenido la Figura 3. 
Figura 3

Relación entre costes bancarios y desempleo en la OCDE entre 2002 y 2013

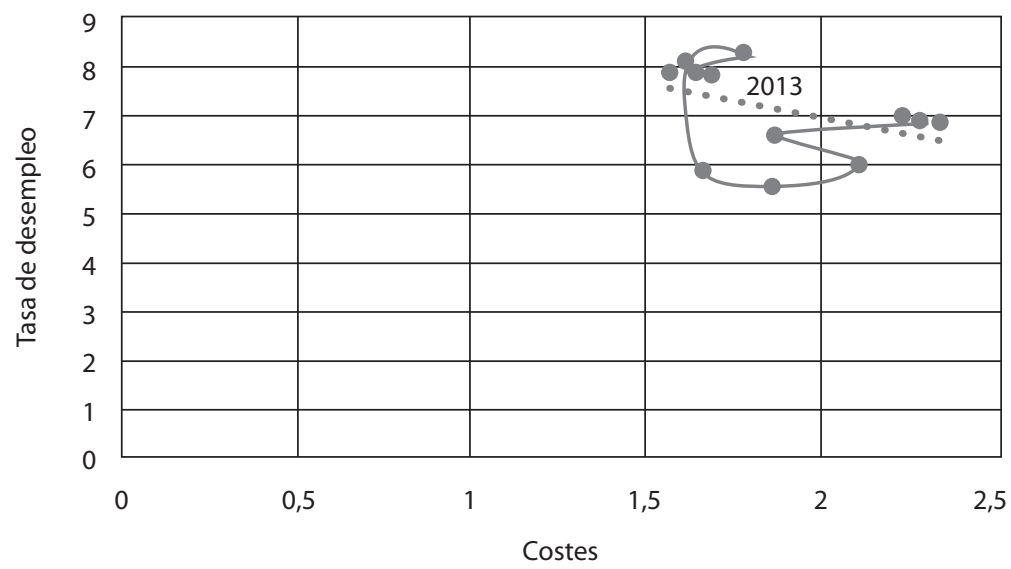

Fuente: OCDE (2017).

En la Figura 3 se puede apreciar claramente una especie de forma de espiral al unir los datos de cada año por orden cronológico. Esta forma recuerda a las espirales encontradas en la relación de no linealidad entre tasa de desempleo y tasa de salario para datos de un mismo país a lo largo del tiempo (Goodwin, 1967), que se especifica a través de una proporcionalidad inversa habitualmente.

Por lo tanto, observando los resultados se deduce que una de las maneras para evitar el excesivo crecimiento de los costes del sector financiero y alcanzar un desarrollo financiero sostenible no se circunscribe únicamente a establecer regulación bancaria y el gravamen de impuestos no distorsionantes como los impuestos sobre servicios financieros o tasas correctoras de externalidades e ineficiencias como los impuestos sobre servicios o transacciones financieras (FMI, 2010), sino que hay que tener en cuenta también otras medidas para diluir el, en ocasiones, mal funcionamiento de la banca. Posibles alternativas a las anteriores serían la rectificación de la política monetaria de forma que siga criterios (reglas) económicos (Taylor, 2009 y Peña, 2017), el 
establecimiento de reformas bancarias que eliminen el vínculo entre el sector bancario y la desigualdad económica (Perugini et al., 2015) o esterilizar políticas activas y pasivas de empleo para evitar el efecto no deseado sobre la eficiencia bancaria, como hemos visto en este artículo.

Así, haría falta una reforma del sistema bancario y del mercado laboral para eliminar este tipo de cadenas de transmisión de la economía real a la financiera, para intentar impedir que una crisis financiera se convierta en crisis económica.

Por lo tanto, consideramos que el análisis de las crisis generadas por el excesivo crédito puede compararse con el tratamiento de un paciente. En muchas ocasiones, el seguimiento de criterios no económicos (por ejemplo, no seguir reglas de política monetaria como la de Taylor, que se basa en variables como la inflación) se puede considerar como la "enfermedad" que lleva al mal funcionamiento del sector financiero $y$, por lo tanto, a la crisis económica. El gravamen de los servicios y transacciones financieras se pueden ver como el "tratamiento" que permite corregir las distorsiones del sector financiero o las externalidades negativas generadas por una incorrecta política monetaria o de control bancario. En cambio, el establecimiento de regulación bancaria para robustecer a los bancos frente a las crisis, eliminando el vínculo entre política monetaria y crédito (quizás también fuera necesario regular y vigilar la política monetaria de los bancos centrales que, a pesar de ser organismos independientes, deberían seguir reglas económicas), constituye un ejemplo de "prevención" de la enfermedad y sus "secuelas" (p.ej., ineficiencia bancaria, crisis financieras y económicas). Otros ejemplos de prevención serían la eliminación de otros canales de transmisión de la economía financiera a la real, como los vinculados a desigualdad económica o, como se ha visto en este trabajo, al desempleo.

\section{CONCLUSIONES}

En este trabajo se analiza el impacto del desempleo sobre los costes del sector financiero utilizando un panel no balanceado de los 35 países miembros de la OCDE durante el periodo de 1996 a 2014. Para ello se utiliza la metodología GMM, después de haber realizado un análisis descriptivo de los datos en el que 
se observa una relación negativa entre la variable dependiente y la variable objetivo.

Se obtiene una corroboración de la significatividad del impacto negativo del desempleo sobre la eficiencia bancaria a través de una relación de proporcionalidad inversa entre ambas variables, confirmando que la tasa de desempleo influye en el desarrollo financiero.

\section{REFERENCIAS BIBLIOGRÁFICAS}

Acemoglu, D. (2001). Credit market imperfections and persistent unemployment. European Economic Review, 45(4-6): 665-679.

Allen, F., Carletti, E., Cull, R., Qian, J., Senbet, L. y Valenzuela, P. (2014). The African Financial Development and Financial Inclusion Gaps. Journal of African economies, 23(5), 614642.

Aliero, H. M., Ibrahim, S. S., \& Shuaibu, M. (2013). An Empirical Investigation into the Relationship between Financial Sector Development and Unemployment in Nigeria. Asian Economic and Financial Review, 3(10), 1361.

Arellano, M., y Bond. S. (1991): Some tests of specification for panel data: Monte Carlo evidence and an application to employment equations. Review of Economic Studies, 58, 277297.

Arellano, M. y Bover, O. (1995). Another look at the instrumental variable estimation of error-components models. Journal of Econometrics, 68, 29-51.

Asongu, S. (2014). Financial development dynamic thresholds of financial globalization. Journal of Economic Studies, 41(2), 166-195.

Ayadi, R., Arbak, E., Ben Naceur, S. y De groen, W. P. (2013). Determinants of Financial Development across the Mediterranean. MEDPRO Technical Report No. 29, European Commission.

Bahadir, B. y Valev, N. (2015). Financial Development Convergence. Journal of Banking \& Finance, 56, 61-71.

Banco Mundial (2017). https://datos.bancomundial.org/ 
Belot, M. y Van Ours, J.C. (2001). Unemployment and labor market institutions. an empirical analysis. Journal of the Japanese and International Economies, 15(4), 403-418.

Blanchard, O. J., Jaumotte, F. y loungani, P. (2014). Labor market policies and IMF advice in advanced economies during the Great Recession. IZA Journal of Labor Policy, 3(1), 2.

Blundell, R. y Bond, S. (1998). Initial conditions and moment restrictions in dynamic panel data models. Journal of Econometrics, 87, 115-143.

Blundell, R. y Bond, S. (2000). GMM Estimation with Persistent Panel Data: An Application to Production Functions. Econometric Reviews, 19(3), 321-40.

Boyd, J.H., Levine, R. y Smith, B.D. (2001). The impact of inflation on financial sector performance. Journal of Monetary Economics, 47(2): 221-248.

Caballero, R.J. y Krishnamurthy, A. (2004). Fiscal policy and financial depth. NBER Working Paper No. 10532, National Bureau of Economic Research, Cambridge, MA.

Cecchetti, S. G. y Kharroubi, E. (2012). Reassessing the impact of finance on growth. BIS Working Papers, n 381, 2012.

Christensen, J. (2004). Domestic debt markets in Sub-Saharan Africa. IMF Working Papers 04/46. International Monetary Fund, Washington, D.C.

Dehejia, R. y Gupta, N. (2016). Financial Development and Occupational Choice. Mimeo.

Demirgüç-kunt, A., Córdova, E.L., Pería, M.S.M. y Woodruff, C. (2011). Remittances and banking sector breadth and depth: Evidence from Mexico. Journal of Development Economics, 95(2). 229-241.

Detragiache, E., Gupta, P. y Tressel, T. (2005). Finance in lower income countries: an empirical exploration. IMF Working Paper No. 05/167, International Monetary Fund, Washington, D.C.

Dromel, N.L., Kolakez, E. y Lehmann, E. (2010). Credit constraints and persistence of unemployment. Labour Economics, 17, 823-834. 
Fondo Monetario Internacional (FMI, 2010). A Fair and Substantial Contribution by the Financial Sector, Final Report for the G-20.

Goodwin, R.M. (1967). A growth cycle, en C.H. Feinstein (ed). Socialism, capitalism and economic growth. London, MacMillan, pp. 165-170.

Huang, Y. (2010). Determinants of Financial Development, ed. New York: Palgrave Macmillan.

Huizinga, H. (2002). A European VAT on financial services? Economic Policy, 17(35), 497-534.

Ibrahim, S.S. y Aliero, H.M. (2012). Does access to finance reduce poverty? Evidence from rural areas of katsina state. Mediterranean Journal of Social Science, 3(4), 575-581.

Khan, M.S.; Senhadji, A.S.; y Smith, B. D. (2006). Inflation and financial depth. Macroeconomic Dynamics, 10, 165-182.

King, R. G. y Levine, R. (1993). Finance and growth. Schumpeter Might Be Right. Quarterly. Journal of Economics, 108(3), 71737.

Nickell, S., Nunziata, L. y Ochel, W. (2005). Unemployment in the OECD since the 1960s. What do we know? The Economic Journal, 115(500), 1-27.

OCDE (2017). OCDE Database (https://data.oecd.org/).

Ogbeide, F. I., Kanwanye, H. y Kadiri, S. (2015). The Determinants of Unemployment and the Question of Inclusive Growth in Nigeria: Do Resource Dependence, Government Expenditure and Financial Development Matter? Montenegrin Journal of Economics, 11(2), 49.

Peña, G. (2017). Money, Lending and Banking Crises. Economic Papers: A journal of applied economics and policy.

Perugini, C., Hölscher, J., \& Collie, S. (2015). Inequality, credit and financial crises. Cambridge Journal of Economics, 40(1): 227-257.

Rajan, R.G. y Zingales, L. (2003). The Great Reversals: The Politics of Financial Development in the Twentieth Century. Journal of Financial Economics, 69(1). 5-50.

Taylor, J. B. (2009). The financial crisis and the policy responses: An empirical analysis of what went wrong. NBER Working 
Paper No. 14631, National Bureau of Economic Research, MA: Cambridge.

Schumpeter, J. A. (1911). The theory of economic development. An inquiry into profits, capital, credit, interest, and the Business cycle. MA: Harvard University Press.

Windmeijer, F. (2005). A finite sample correction for the variance of linear efficient two-step GMM estimators. Journal of Econometrics, 126(1). 25-51. 